\title{
Fatores associados à úlcera por pressão em pacientes internados nos Centros de Terapia Intensiva de Adultos
}

\author{
FACTORS ASSOCIATED TO PRESSURE ULCERS IN PATIENTS AT ADULT INTENSIVE \\ CARE UNITS
}

FACTORES ASOCIADOS A LA ÚLCERA POR PRESIÓN EN PACIENTES INTERNADOS
EN UNIDADES DE TERAPIA INTENSIVA DE ADULTOS

Flávia Sampaio Latini Gomes ${ }^{1}$, Marisa Antonini Ribeiro Bastos², Fernanda Penido Matozinhos ${ }^{3}$, Hanrieti Rotelli Temponi ${ }^{4}$, Gustavo Velásquez-Meléndez ${ }^{5}$

\section{RESUMO}

Trata-se de um estudo seccional analítico com o objetivo de estimar a ocorrência de úlceras por pressão e seus fatores associados em CTIs de adultos, em Belo Horizonte. A amostra foi constituída por 142 pacientes, com 18 anos ou mais, internados até as $24 \mathrm{~h}$ do dia anterior à coleta de dados, em 316 leitos, distribuídos em 22 CTIs. A presença de pelo menos uma úlcera por pressão, e por paciente, foi de $35,2 \%$ (IC $95 \%=27,4-47,7)$. Das 99 úlceras identificadas, constatou-se que foram mais frequentes nas regiões sacral $(36,0 \%)$ e calcânea $(22,0 \%)$. Observou-se que presença de sepses (OR = 6,04; IC 95\% = 1,09-33,53), tempo de internação $\geq 10$ dias $(O R=7,61$; IC $95 \%=2,92-19,82)$ e risco alto e elevado na classificação da escala de Braden (OR = 4,96; IC $95 \%=1,50-16,50$ ) foram fatores independentes e significativamente associados à presença de úlcera por pressão. Conclui-se que sepses, tempo de internação e risco alto e elevado na classificação da escala de Braden são fatores potencialmente associados à formação de úlceras em pacientes acamados.

\begin{abstract}
The objective of this a cross-sectional analytical study was to estimate the occurrence of pressure ulcers and their associated factors in adult ICUs in the city of Belo Horizonte, Brazil. The sample consisted of 142 patients aged 18 or older, admitted before midnight of the day prior to data collection, from a total of 316 beds distributed in $22 \mathrm{ICU}$ s. Presence of at least one pressure ulcer per patient was $35.2 \%(\mathrm{Cl}$ $95 \%=27.4-47.7)$. Of the 99 ulcers identified, the ones in the sacral region were most frequent $(36.0 \%)$, followed by those in the calcaneus $(22.0 \%)$. We observed that the presence of sepsis $(\mathrm{OR}=6.04, \mathrm{Cl} 95 \%=1.09$ 33.53 ), period of stay $\geq 10$ days ( $O R=7.61$, $\mathrm{Cl} 95 \%=2.92-19.82)$ and being high risk and very high-risk in the Braden scale $(\mathrm{OR}=$ 4.96 , $\mathrm{Cl} 95 \%=1.50-16.50$ ) were independent factors significantly associated with the presence of pressure ulcers. Results suggest that sepsis, length of stay, and having high and very high risk" in the Braden scale are factors potentially associated to the development of ulcers in bedridden patients.
\end{abstract}

\section{KEY WORDS}

Pressure ulcer.

Risk factors.

Intensive Care Units.

Epidemiology.

\begin{abstract}
RESUMEN
Se trata de un estudio seccional analítico que tuvo como objetivo estimar la ocurrencia de úlceras por presión y sus factores asociados en UTIs de adultos en Belo Horizonte-MG-Brasil. La muestra fue constituida por 142 pacientes, con edad de 18 años o superior, internados hasta las $24 \mathrm{hs}$. del día anterior a la recolección de datos, en 316 lechos distribuidos en 22 UTIs. La presencia de por lo menos una úlcera por presión por paciente fue de $35,2 \%$ (IC 95\% - 27,4$47,7)$. De las 99 úlceras identificadas, se constató que fueron más frecuentes en las regiones sacra $(36,0 \%)$ y calcánea $(22,0 \%)$. Se observó que la presencia de sepsis (OR = 6,04; IC 95\% = 1,09-33,53), tiempo de internación $\geq 10$ días (OR = 7,61; IC 95\% = $2,92-19,82)$ y riesgo alto y elevado en la clasificación de la escala de Braden (OR $=4,96$; IC $95 \%=1,50-16,50$ ) fueron factores independientes y significativamente asociados a la presencia de úlcera por presión. Se concluye en que la sepsis, tiempo de internación y riesgo alto y elevado en la clasificación de la escala de Braden son factores potencialmente asociados a la formación de úlceras en pacientes acostados.
\end{abstract}

\section{DESCRIPTORES \\ Úlcera por presión. \\ Factores de riesgo. \\ Unidades de Terapia Intensiva. \\ Epidemiología.}

${ }_{1}^{1}$ Professora Adjunta do Departamento de Enfermagem Básica da Escola de Enfermagem da Universidade Federal de Minas Gerais. Belo Horizonte, MG, Brasil. latini@ufmg.br ${ }^{2}$ Professora Titular da Faculdade de Ciências da Saúde da Fundação Mineira de Educação e Cultura. Belo Horizonte, MG, Brasil. mbastos@fcs.fumec.br ${ }^{3}$ Graduanda de Enfermagem da Escola de Enfermagem da Universidade Federal de Minas Gerais. Belo Horizonte, MG, Brasil. nandapenido@hotmail.com ${ }^{4}$ Graduanda de Enfermagem da Escola de Enfermagem da Universidade Federal de Minas Gerais. Belo Horizonte, MG, Brasil. hanrietirari@gmail.com ${ }^{5}$ Professor Titular do Departamento de Enfermagem Materno Infantil e Saúde Pública da Escola de Enfermagem da Universidade Federal de Minas Gerais. Belo Horizonte, MG, Brasil. guveme@ufmg.br

$17 \begin{aligned} & \text { Rev Esc Enferm USP } \text { Recebido: } 16 / 10 / 2009 \\ & \text { 2010; 44(4):1070-6 } \text { Aprovado: 26/03/2010 } \\ & \text { www.ee.usp.br/reeuspl } \text { Português/Inglês } \\ & \text { www.scielo.br/reeusp }\end{aligned}$




\section{INTRODUÇÃO}

As úlceras por pressão (UP) são, geralmente, definidas como áreas localizadas de necrose celular que ocorrem sobre proeminências ósseas expostas à pressão por um período suficiente de tempo para causar isquemia tecidual. $O$ desenvolvimento das úlceras por pressão é multifatorial, mas o principal fator é a pressão exercida sobre um capilar, entre o arcabouço ósseo e uma superfície, colabando-o e ocasionando a necrose tissular. A prevalência de úlcera por pressão entre pacientes adultos hospitalizados pode variar de 3 a $14 \%^{(1)}$.

Nos Estados Unidos, em uma pesquisa nacional realizada em 1999 sobre prevalência de úlcera por pressão, 42.817 pacientes hospitalizados foram avaliados, determinando a seguinte distribuição por clínica: médica (28\%), cirúrgica (13\%), semi-intensiva (9\%), ortopédica (7\%) e terapia intensiva (7\%). A prevalência global de pacientes com úlcera por pressão nesse estudo foi $14,8 \%$, sendo que as unidades de terapia intensiva obtiveram os maiores valores $(21,5 \%)^{(2)}$.

Em estudo conduzido por três meses consecutivos em um hospital universitário no Brasil(3) foi identificada a incidência de úlcera por pressão de $39,8 \%$, variando de acordo com a unidade hospitalar estudada, sendo de $41,0 \%$ no centro de terapia intensiva. Para nosso conhecimento, existem poucos estudos que abordam este tema nos serviços de saúde do Brasil.

\section{OBJETIVO}

O presente estudo teve como objetivo estimar a ocorrência de úlcera por pressão e seus fatores associados, nos Centros de Terapia Intensiva (CTI) de adultos, em Belo Horizonte.

\section{MÉTODO}

Trata-se de um estudo seccional analítico. O estudo transversal, de corte ou seccional descritivo refere-se a uma estimativa de prevalência ou ocorrência de um determinado evento. Nos estudos transversais, de corte ou seccionais analíticos, alem da ocorrência, procura-se verificar se eventos estão associados. No entanto, sabe-se que as conclusões obtidas pelas análises desse estudo restringem-se a relações de associação e não de causalidade ${ }^{(4)}$.

A população foi constituída de pacientes com 18 anos ou mais, que foram internados até as $24 \mathrm{~h}$ do dia anterior à coleta de dados, a partir de um universo de 316 leitos distribuídos em 22 CTIs, de 15 hospitais públicos e privados de Belo Horizonte, Minas Gerais, que também atendiam à Saúde Suplementar.

Utilizou-se o programa Epi Info 6.0 para o sorteio e o cálculo do tamanho amostral com os seguintes parâmetros: prevalência esperada de úlcera por pressão de $30 \%$, erro de $6 \%$, nível de confiança de $95 \%$ e poder de $80 \%$, considerando um universo de 316 leitos disponíveis para o estudo. Segundo esses parâmetros, a amostra deveria ser constituída de 134 indivíduos. Acrescentou-se 40\% para possíveis perdas e análises com mais de duas variáveis, donde se obteve estimativa de 187 pacientes.

Dos 187 leitos sorteados que compunham a amostra, no momento da coleta de dados, 33 leitos estavam vagos. Dentre os 154 pacientes restantes, doze não foram disponíveis, uma vez que cinco se recusaram a participar da coleta de dados, três estavam internados há menos de 24 horas, dois encontravam-se impedidos de serem manipulados e avaliados e dois eram menores de 18 anos. Sendo assim, a amostra final foi constituída por 142 pacientes.

A coleta de dados foi realizada no mês de julho de 2007, em um único dia. $O$ instrumento de coleta de dados foi um formulário contendo questões fechadas e abertas referentes às informações da instituição, da pessoa, seu perfil sociodemográfico e seus dados clínicos; da pontuação na Escala de Risco de Braden, do número, estadiamento e localização de úlceras por pressão, além de medidas preventivas utilizadas.

Outras variáveis contempladas no formulário de coleta de dados relacionadas às medidas preventivas foram: uso de superfície preventiva, uso de óleo ou creme hidratante, manutenção da pele limpa e seca, reposicionamento, controle de incontinência e suporte ou suplementação nutricional.

Estes dados foram anotados conforme os registros médicos e de enfermagem contidos no prontuário do paciente ou segundo informações cedidas pela equipe profissional da instituição. Contudo, não foi possível avaliar a implementação e cumprimento de tais medidas, devido ao tempo exíguo da coleta de dados.

As variáveis de estudo foram: idade, sexo, cor de pele, tempo de internação, tempo de internação no CTI, doença de base, medicamentos em uso contínuo, tabagismo, índice de massa corporal, número de úlceras por pressão, localização das úlceras, estadiamento. As variáveis percepção sensorial, umidade, atividade, mobilidade, nutrição e fricção e cisalhamento foram utilizadas segundo a Escala de Braden.

Os dados foram processados e analisados por meio do programa Statistical Package for Social Science (SPSS) - versão 15.0. A associação entre variáveis dependentes e presença de úlcera por pressão foi testada por meio do cálculo do odds ratio (OR) e seus intervalos de confiança de 95\% (IC 95\%), empregando-se a técnica de regressão logística multivariada.

A realização do estudo foi aprovada pelo Comitê de Ética em Pesquisa da Faculdade FUMEC (Parecer no 265/2007) e, aos pacientes ou responsáveis, foram solicitadas sua aprovação para participarem do estudo, leitura e assinatura do Termo de Consentimento Livre e Esclarecido. 


\section{RESULTADOS}

A caracterização da amostra foi realizada segundo sexo, idade, cor de pele, índice de massa corpórea (IMC), tabagismo, tempo total de internação, tempo de internação no CTI e categoria do convênio de saúde, descrita na Tabela 1.

Tabela 1- Caracterização dos pacientes pertencentes à amostra. Belo Horizonte - 2007

\begin{tabular}{|c|c|c|c|c|}
\hline Variáveis & $\mathbf{N}$ & $\%$ & Média & DP \\
\hline \multicolumn{5}{|l|}{ Sexo } \\
\hline Masculino & 75 & 53,0 & & \\
\hline Feminino & 67 & 47,0 & & \\
\hline Total & 142 & 100,0 & & \\
\hline \multicolumn{5}{|c|}{ Faixa etária (anos) } \\
\hline $18-31$ & 5 & 3,0 & & \\
\hline $32-45$ & 12 & 9,0 & & \\
\hline $46-59$ & 35 & 25,0 & & \\
\hline 60 ou mais & 90 & 63,0 & & \\
\hline Total & 142 & 100,0 & 64,0 & 16,7 \\
\hline \multicolumn{5}{|l|}{ Cor de pele } \\
\hline Branca & 90 & 65,0 & & \\
\hline Parda & 34 & 25,0 & & \\
\hline Negra & 14 & 10,0 & & \\
\hline Amarela & 0 & - & & \\
\hline Vermelha & 0 & - & & \\
\hline Total & 138 & 100,0 & & \\
\hline \multicolumn{5}{|l|}{ IMC $\left(\mathrm{Kg} / \mathrm{m}^{2}\right)$} \\
\hline$<18,5$ & 3 & 5,0 & & \\
\hline $18,5-24,9$ & 20 & 33,0 & & \\
\hline $25,0-29,9$ & 37 & 62,0 & & \\
\hline$>30,0$ & 0 & - & & \\
\hline Total & 60 & 100,0 & & \\
\hline \multicolumn{5}{|l|}{ Tabagismo } \\
\hline Não & 49 & 55,0 & & \\
\hline Sim & 13 & 14,0 & & \\
\hline Ex-fumante & 28 & 31,0 & & \\
\hline Total & 90 & 100,0 & & \\
\hline \multicolumn{5}{|c|}{ Tempo total de internação (dias) } \\
\hline $1-10$ & 77 & 54,0 & & \\
\hline $11-20$ & 25 & 18,0 & & \\
\hline $21-30$ & 11 & 8,0 & & \\
\hline $31-40$ & 9 & 6,0 & & \\
\hline $41-50$ & 7 & 5,0 & & \\
\hline$>50$ & 13 & 9,0 & & \\
\hline Total & 142 & 100,0 & 18,0 & 26,16 \\
\hline \multicolumn{5}{|c|}{ Tempo de internação no CTI (dias) } \\
\hline$\leq 10$ & 99 & 70,0 & & \\
\hline $11-20$ & 19 & 13,0 & & \\
\hline $21-30$ & 12 & 9,0 & & \\
\hline $31-40$ & 6 & 4,0 & & \\
\hline $41-50$ & 2 & 1,0 & & \\
\hline$>50$ & 4 & 3,0 & & \\
\hline Total & 142 & 100,0 & 13,0 & 13,12 \\
\hline \multicolumn{5}{|c|}{ Convênio de saúde } \\
\hline SUS & 39 & 28,0 & & \\
\hline Suplementar & 98 & 71,0 & & \\
\hline Particular & 2 & 1,0 & & \\
\hline Total & 139 & 100,0 & & \\
\hline
\end{tabular}

Deve-se esclarecer que os totais dos números de pacientes podem variar, devido às diferentes taxas de não respostas, para as variáveis estudadas.

Em relação às instituições participantes do estudo, em um dos hospitais todos os leitos (02) do CTI sorteados encontravam-se desocupados, no momento da coleta de dados. Dos leitos sorteados, 67,0\% (04) encontravam-se vazios em outro hospital e 44,0\% (08 e 04) em outros dois. Em quatro hospitais participantes, todos os leitos sorteados estavam ocupados.

A ocorrência de pelo menos uma úlcera por pressão por paciente foi de 35,2\% (IC 95\%: 27,4-47,7). Portanto, espera-se que 111 pacientes (variando entre 87 a 151) apresentem pelo menos uma úlcera por pressão no total de leitos disponíveis em CTIs das quinze instituições avaliadas (316 leitos).

Dos 50 pacientes portadores de úlceras por pressão, 27 $(19,0 \%)$ apresentavam uma única úlcera, 11 pacientes $(7,7 \%)$ duas úlceras, 12 pacientes $(8,5 \%)$ três ou mais úlceras. Dentre estes últimos, em apenas um deles foram identificadas 12 úlceras. Entre os 142 pacientes analisados, 92 $(64,8 \%)$ não apresentavam úlcera por pressão, no momento da coleta de dados.

Constatou-se que não houve diferença significativa entre ocorrência de úlcera por pressão segundo o sexo ( $p=$ $0,27)-40,0 \%$ entre homens e $30,0 \%$ entre mulheres - e segundo a cor de pele $(p=0,53)$ - parda $(38,0 \%)$, branca $(35,0 \%)$ e negra $(21,0 \%)$. A ocorrência foi maior entre os indivíduos de 45 a 59 anos $(71,4 \%)$, comparada às faixas etárias de 60 anos ou mais (40,0\%), 32 a 45 anos (27,3\%) e 18 a 31 anos (20,0\%). Entretanto, as diferenças não foram estatisticamente significativas $(p=0,45)$.

A ocorrência de úlcera por pressão segundo categorias de IMC ( $n=60$ pacientes) foi de 50,0\% nos eutróficos e de $16,2 \%$ nos pacientes com sobrepeso. Não foi constatada ocorrência de úlcera por pressão em nenhum dos três pacientes desnutridos. Salienta-se que não foram observadas diferenças estatísticas das freqüências de úlcera por pressão segundo estado nutricional $(p=0,179)$.

Em relação ao hábito de fumar, em 90 pacientes, a prevalência de úlcera por pressão entre os fumantes foi de $42,6 \%$, entre os não-fumantes foi de $30,6 \%$ e de $14,3 \%$ entre os ex-fumantes. Não houve associação estatística entre essas variáveis $(p=0,085)$.

De acordo com o tempo total de internação dos pacientes nos hospitais que compuseram a amostra, a prevalência de úlcera por pressão foi progressivamente maior naqueles com mais de 10 dias de permanência, com diferenças estatisticamente significativas $(p=0,00)$.

Ao comparar com o tempo de internação dos pacientes nos CTIs, a freqüência acumulada de úlceras por pressão foi maior entre aqueles grupos com tempo de permanência superior a 10 dias. Todos os pacientes que estavam no 
CTI há mais de 50 dias eram portadores de úlcera por pressão. A relação entre tempo de internação no CTI e presen- ça de úlcera por pressão foi estatisticamente significativa $(p=0,00)$, conforme Tabela 2 .

Tabela 2 - Prevalência de úlcera por pressão segundo o tempo total de internação do paciente no hospital e o tempo de internação do paciente no CTI - Belo Horizonte - 2007

\begin{tabular}{|c|c|c|c|c|c|c|}
\hline \multirow{3}{*}{$\begin{array}{l}\text { Tempo de } \\
\text { internação } \\
\text { (em dias) }\end{array}$} & \multicolumn{6}{|c|}{ Úlcera por pressão } \\
\hline & \multicolumn{2}{|c|}{ Sim } & \multicolumn{2}{|c|}{ Não } & \multicolumn{2}{|c|}{ Total } \\
\hline & $\mathbf{N}$ & $\%$ & $\mathbf{N}$ & $\%$ & $\mathbf{N}$ & $\%$ \\
\hline \multicolumn{7}{|l|}{ No hospital } \\
\hline $1-10$ & 9 & 11,7 & 68 & 88,3 & 77 & 54,2 \\
\hline $11-20$ & 14 & 56,0 & 11 & 44,0 & 25 & 17,6 \\
\hline $21-30$ & 6 & 54,5 & 5 & 45,5 & 11 & 7,7 \\
\hline $31-40$ & 5 & 55,6 & 4 & 44,4 & 9 & 6,3 \\
\hline $41-50$ & 5 & 71,4 & 2 & 28,6 & 7 & 4,9 \\
\hline$>50$ & 11 & 84,6 & 2 & 15,4 & 13 & 9,2 \\
\hline Total & 50 & 35,2 & 92 & 64,8 & 142 & 100,0 \\
\hline \multicolumn{7}{|l|}{ No CTI } \\
\hline $1-10$ & 22 & 28,6 & 77 & 71,4 & 99 & 69,7 \\
\hline $11-20$ & 11 & 59,9 & 8 & 42,1 & 19 & 13,5 \\
\hline $21-30$ & 9 & 75,0 & 3 & 25,0 & 12 & 8,4 \\
\hline $31-40$ & 3 & 50,0 & 3 & 50,0 & 6 & 4,2 \\
\hline $41-50$ & 1 & 50,0 & 1 & 50,0 & 2 & 1,4 \\
\hline$>50$ & 4 & 100,0 & 0 & 0 & 4 & 2,8 \\
\hline Total & 50 & 35,2 & 92 & 64,8 & 142 & 100,0 \\
\hline
\end{tabular}

A prevalência de úlcera por pressão não foi estatisticamente diferente $(p=0,52)$ segundo o plano de saúde. Entre os pacientes que eram atendidos pelo Sistema Único de Saúde (SUS), 38,5\% deles apresentavam úlceras. Dentre aqueles que possuíam convênio de saúde suplementar, $34,7 \%$ eram portadores de úlceras. Apenas dois pacientes estavam internados com recursos próprios, não apresentavam úlceras.
As doenças de base registradas nos formulários foram classificadas de acordo com o sistema acometido e aquelas que pertenciam aos sub-grupos neurológico (13), digestório (13), osteomuscular (6) e geniturinário (1) foram agrupadas no item outros. Segundo a Tabela 3, a maior prevalência de úlcera por pressão ocorreu entre pacientes portadores de sepse, seguida de doenças do sistema respiratório, como insuficiência respiratória pulmonar aguda, trombo-embolismo pulmonar e pneumonia, cuja associação foi significativa $(p=0,00)$.

Tabela 3 - Prevalência de úlcera por pressão conforme a doença de base do paciente - Belo Horizonte - 2007

\begin{tabular}{|c|c|c|c|c|c|c|}
\hline \multirow{3}{*}{ Doença de base } & \multicolumn{6}{|c|}{ Úlcera por pressão } \\
\hline & \multicolumn{2}{|c|}{ Sim } & \multicolumn{2}{|c|}{ Não } & \multicolumn{2}{|c|}{ Total } \\
\hline & $\mathbf{N}$ & $\%$ & $\mathbf{N}$ & $\%$ & $\mathbf{N}$ & $\%$ \\
\hline \multicolumn{7}{|l|}{ Sistema } \\
\hline Circulatório & 9 & 16,6 & 45 & 83,4 & 54 & 38,0 \\
\hline Respiratório & 24 & 54,5 & 20 & 45,5 & 44 & 31,0 \\
\hline Outros & 10 & 30,3 & 23 & 69,7 & 33 & 23,3 \\
\hline Sepse & 7 & 63,6 & 4 & 36,4 & 11 & 7,7 \\
\hline TOTAL & 50 & 35,2 & 92 & 64,8 & 142 & 100,0 \\
\hline
\end{tabular}

Assim como havia mais de uma doença de base relatada nos formulários de coleta de dados, havia também mais de um medicamento em uso contínuo pelos pacientes. As classes de medicamentos mais prescritas foram a dos broncodilatadores, analgésicos, antiinflamatórios esteroidais e não-esteroidais e anti-coagulantes ( $n=141)$, seguidos de diuréticos $(n=140)$, anti-hipertensivos ( $n=139)$ e cardiotônicos ( $n=137$ ). No item outros foram descritos medicamentos como anti-depressivos, ansiolíticos, mucolíticos, hipoglicemiantes (insulina), protetores de mucosa gástrica, antibióticos, hormônio tireioideano, vitaminas, antiarrítmicos e soluções endovenosas.

Observa-se associação significativa entre uso de broncodilatadores e úlcera por pressão, sendo $p=0,00$. O uso dos demais medicamentos não foi associado significativamente à presença de úlcera por pressão. 
Tabela 4 - Prevalência de úlcera por pressão conforme o medicamento em uso contínuo pelo paciente - Belo Horizonte - 2007

\begin{tabular}{lccccc}
\hline \multirow{2}{*}{ Medicamentos } & \multicolumn{5}{c}{ Úlcera por pressão } \\
\cline { 2 - 6 } & \multicolumn{3}{c}{ Sim } & \multicolumn{3}{c}{ Não } & p \\
\cline { 2 - 6 } N & \% & N & \% & \\
\hline Anti-hipertensivos & 27 & 29,9 & 50 & 70,1 & 0,94 \\
Cardiotônicos & 12 & 31,6 & 26 & 68,4 & 0,58 \\
Broncodilatadores & 28 & 60,9 & 18 & 39,1 & 0,00 \\
Analgésicos & 25 & 40,3 & 37 & 59,7 & 0,37 \\
Antiinflamatórios & 18 & 43,9 & 23 & 56,1 & 0,25 \\
Diuréticos & 24 & 43,6 & 31 & 56,4 & 0,16 \\
Anti-coagulantes & 32 & 36,4 & 56 & 63,6 & 0,91 \\
Outros & 44 & 37,9 & 72 & 62,1 & 0,27 \\
\hline
\end{tabular}

Nota: $(n=50)$

A localização das úlceras por pressão foi registrada em todos os formulários; entretanto, como alguns pacientes eram portadores de mais de uma úlcera, o total foi de 99 úlceras, distribuídas nas seguintes regiões: lóbulos das oreIhas $(3,0 \%)$, occipital $(1,0 \%)$, escápulas $(3,0 \%)$, processo espinhoso $(2,0 \%)$, ílio $(6,0 \%)$, sacro $(36,0 \%)$, cotovelos $(3,0 \%)$, trocânteres $(9,0 \%)$, tuberosidades isquiáticas $(4,0 \%)$, tíbias $(4,0 \%)$, maléolos $(4,0 \%)$, podáctilos $(2,0 \%)$ e calcâneos $(22,0 \%)$.

As 99 úlceras foram avaliadas de acordo com seu estágio, de I a IV. As úlceras de estágio I representavam 25,0\%; de estágio II, 57,0\%; de estágio III, 9,0\% e de estágio IV, $6,0 \%$. Devido ao fato de estarem totalmente recobertas por necrose, três das feridas $(3,0 \%)$ não foram avaliadas quanto ao seu estadiamento.

Por meio da técnica de regressão logística multivariada, observou-se que a presença de sepses (OR = 6,04; IC 95\% = $1,09-33,53)$, tempo de internação $\geq 10$ dias (OR = 7,61; IC $95 \%=2,92-19,82$ ) e risco alto e elevado na classificação da escala de Braden (OR = 4,96; IC 95\% = 1,50-16,50) foram fatores independentes e significativamente associados à presença de úlcera por pressão.

Tabela 5 - Fatores associados à úlcera por pressão, odds ratio e intervalo de confiança de 95\% - Belo Horizonte - 2007

\begin{tabular}{lcc}
\hline Variáveis & OR & IC 95\% \\
\hline Sepses & 6,04 & $1,09-33,53$ \\
Tempo de internação $>10$ dias & 7,61 & $2,92-19,82$ \\
Risco alto e elevado na escala de Braden & 4,96 & $1,50-16,50$ \\
\hline
\end{tabular}

O valor médio dos escores da escala de Braden nos 140 pacientes foi 13,86; segundo o sexo, o valor médio foi 14,13 $(\mathrm{dp}=4,63)$ para pessoas do sexo masculino e 13,56 (dp = $4,91)$ para pessoas do sexo feminino, sem diferenças estatísticas $(p=0,48)$. Segundo a idade, os escores médios foram 15,22 (dp $=4,41)$ para pacientes menores de 60 anos e $13,03(\mathrm{dp}=4,79$ ) para pacientes com 60 anos ou mais, sendo essa diferença estatisticamente significativa $(p=0,00)$.

\section{DISCUSSÃO}

Foi realizada a caracterização da amostra, composta por 142 pacientes, além da determinação da prevalência de úlceras por pressão em Centros de Terapia Intensiva e estabelecimento dos fatores que estariam associados ao desenvolvimento das úlceras.

A descrição da amostra em relação ao índice de massa corpórea (IMC) e hábito de fumar foi prejudicada, em decorrência de subnotificação. A variável IMC foi composta de registros de peso e altura de apenas 60 pacientes. Podese justificar tal achado pela impossibilidade de encontrar tais dados no prontuário do paciente e dificuldade de realização destas medidas no ambiente dos CTIs.

Por exemplo, quanto ao hábito de fumar, muitos dos prontuários de pacientes não continham informações dessa variável ou o próprio paciente ou acompanhante não tinham possibilidade de dar a informação. Sendo assim, 52 formulários não foram preenchidos nos campos reservados a esta variável.

Em relação à ocorrência de úlcera por pressão encontrada nos CTIs pertencentes à amostra, nota-se uma taxa superior àquelas descritas na literatura, entretanto, no Brasil, existem ainda poucos estudos sobre o assunto. Vale acrescentar a ocorrência de úlcera por pressão encontrada em pesquisas realizadas em outros países como: Alemanha $(21,1 \%)^{(5)}$, Suécia $(13,2 \%)^{(6)}$ e Singapura $(18,1 \%)^{(7)}$. A prevalência de úlcera por pressão em pessoas adultas em outro estudo foi de $3 \%$ a $11 \%$ em pacientes hospitalizados, podendo chegar a $18 \%$ entre os pacientes hospitalizados restritos ao leito, como nos $\mathrm{CTIS}^{(8)}$. Na assistência hospitalar aguda encontrou-se um percentual de $3 \%$ de ocorrência de úlcera por pressão, e de $45 \%$ em hospitais assistenciais ${ }^{(9)}$.

Isoladamente, em um estudo realizado para identificar a incidência de úlcera por pressão nos pacientes internados no Hospital Universitário da Universidade de São Paulo (USP), obteve-se índice de incidência global de 39,8\%, apresentando variações de acordo com a unidade hospitalar estudada: clínica médica $(42,6 \%)$, centro de terapia intensiva $(41,0 \%)$ e clínica cirúrgica $(39,5 \%)^{(3)}$.

Em outro trabalho, de análise da literatura publicada em periódicos de 1994 a 1998, foram selecionados 47 artigos, considerando as categorias temáticas das diretrizes para previsão e prevenção de úlceras de pressão da Agência Americana para o Cuidado, Política e Pesquisa em Saúde (AHCPR) ${ }^{(10)}$. $\mathrm{O}$ trabalho ressaltava a inexistência de estudos referentes à incidência e prevalência das úlceras por pressão no Brasil, panorama este pouco alterado nos últimos dez anos.

Quanto à localização das úlceras, em estudo realizado no Brasil $^{(3)}$, verificou-se que a região sacra $(33,6 \%)$, dos calcâneos $(24,6 \%)$ e dos glúteos $(23,9 \%)$ foram locais predominantes quanto ao desenvolvimento dessas úlceras, sendo que $53 \%$ do total eram de estágio II; dados que se assemelham com os encontrados neste estudo. 
Neste trabalho, o desenvolvimento de úlcera por pressão não esteve associado à idade. Porém, estudos revelam que a idade avançada é um fator desencadeante dessas úlceras, sendo que $50 \%$ a $70 \%$ dessas lesões desenvolvem-se em pessoas com mais de 70 anos $^{(11-12)}$. Entretanto, encontrou-se associação entre idade ( $\geq 60$ anos) e baixos escores médios da escala de Braden $(13,0)$, ou seja, os idosos encontravam-se em risco para o desenvolvimento dessas úlceras, uma vez que, para esta população considera-se em risco aquela que apresenta pontuação igual ou menor que $17^{(11)}$. Apesar de a população idosa ser mais propensa ao desenvolvimento de úlcera por pressão, a idade não manteve associação no modelo final|(12).

Em relação ao envelhecimento, sabe-se que a diminuição da elasticidade, da textura, da circulação, do nível de reposição celular e do processo de cicatrização da pele, bem como da sensibilidade periférica, são inerentes ao avanço da idade e, indubitavelmente, levam ao aumento do risco de trauma tegumentar ${ }^{(12)}$.

Para investigar a associação de mortalidade e úlcera por pressão, um trabalho foi realizado nos Estados Unidos entre 1990 a 2001, utilizando registros de óbito codificados por causa. Os autores relataram que, aproximadamente, $80 \%$ das mortes associadas às úlceras por pressão ocorreram em pessoas com 75 anos ou mais. Esse índice de mortalidade foi maior entre pessoas da raça negra que branca ${ }^{(13)}$. Quando comparada a ocorrência de úlcera por pressão de acordo com a cor de pele dos pacientes internados nos CTIs de Belo Horizonte, não foram encontradas diferenças.

A respeito da associação entre a variável IMC e ocorrência de úlcera por pressão, embora neste estudo não se tenha obtido em todos os pacientes, sabe-se que pessoas com baixo peso corporal e proeminências ósseas salientes apresentam risco elevado para o desenvolvimento dessas úlceras. Entretanto, as pessoas que apresentam excesso de gordura corporal também estão mais propensas a essas úlceras, devido ao fato de que o tecido adiposo é pouco vascularizado e não é elástico como outros tecidos, tornando-se mais vulnerável à pressão e propenso a romper-se ${ }^{(14)}$.

Vários outros fatores adicionais também podem influenciar o desenvolvimento desse tipo de úlcera, como o tabagismo, porém, não foi encontrada associação desses dois fatores no presente estudo.

Em relação ao tempo de internação, observou-se neste estudo que a prevalência de úlcera por pressão aumentou à medida que o tempo em que o paciente permanecia internado também aumentava. Na análise multivariada, a associação entre tempo de internação maior que dez dias e ocorrência de úlcera por pressão foi muito alta. Ressaltase que na literatura as úlceras por pressão, usualmente, desenvolvem-se dentro das duas primeiras semanas de internação e entre os pacientes idosos, 15,0\% deles desenvolvem úlceras na primeira semana de internação(14).

Freqüentemente, a ocorrência de úlceras por pressão está relacionada à idade avançada, incontinência, debili- dade, paralisia e pacientes inconscientes. As pessoas que apresentam determinadas condições, como doença neurológica ou cardiovascular, desidratação ou má nutrição, anemia, hipotensão, alterações no turgor e elasticidade da pele ou úlcera por pressão prévia, bem aquelas que foram submetidas à anestesia por tempo prolongado ou que sejam pacientes cirúrgicos são mais susceptíveis ${ }^{(11,15)}$.

A condição ser portador de sepse esteve independentemente e fortemente associada à ocorrência de úlcera por pressão neste estudo. Essas infecções acometem pacientes em todas as faixas etárias, com maior freqüência nos extremos de idade e apresentam pior prognóstico em pacientes com idade acima de 50 anos. As manifestações clínicas são inespecíficas e incluem: febre ou hipotermia, taquicardia, taquipnéia e alcalose respiratória, leucocitose ou leucopenia com aumento do número de bastonetes, hipermetabolismo sistêmico e desnutrição, consumo elevado de oxigênio, hipoperfusão sistêmica e acidose metabólica e estado circulatório hiperdinâmico, fatores esses que podem desencadear a formação da úlcera ${ }^{(11)}$.

A hipotensão sistêmica, que caracteriza a evolução clínica da sepse, é indicativa de circulação periférica e perfusão tecidual insuficientes. Nível de pressão arterial diastólica menor que $60 \mathrm{mmHg}$ pode ser indicador de paciente com alto risco para desenvolvimento de úlcera por pressão(11-12).

Os cuidados de enfermagem aos pacientes portadores de úlceras por pressão abrangem intervenções relacionadas ao acompanhamento integral do cliente em risco de adquirir a lesão, por meio da utilização de escalas de predição de risco $^{(16)}$. Neste estudo, optou-se pela utilização da escala de Braden, a mais utilizada e extensivamente testada até o momento, devido a seu bom desempenho em testes de validação e em conformidade com outros estudos sobre o tema ${ }^{(17)}$.

A categorização da pontuação da Escala de Braden se mostrou fortemente associada à presença de úlcera por pressão, sendo mais intensa no grupo de pacientes com valores nas categorias Risco alto e Risco Elevado, e ainda maiores quando o período de internação foi no CTI.

Na tentativa de diminuir os índices de prevalência de úlcera por pressão e melhorar a qualidade de vida do paciente, tem-se proposto métodos de prevenção e agir sistematicamente, prevenindo a formação destas úlceras. Para que haja desenvolvimento de estratégia eficaz de prevenção, as pessoas de risco precisam ser identificadas. A identificação do risco é alcançada por meio da utilização de instrumentos de avaliação específicos para o problema. Entretanto, o valor preditivo de um teste depende da prevalência da condição na população alvo. Assim, em altos níveis de prevalência, aumenta a probabilidade de um teste positivo predizer melhor a condição(18).

Salienta-se a importância da realização de pesquisas para investigação da incidência e prevalência das úlceras por pressão, visando direcionar e uniformizar as condutas, por meio da demonstração da realidade de cada paciente ${ }^{(16)}$. 


\section{CONCLUSÃO}

A ocorrência de pelo menos uma úlcera por pressão por paciente foi de 35,2\%. Salienta-se que na literatura consultada, a prevalência de úlcera por pressão em adultos varia de 3 a $14 \%$, podendo chegar até $21 \%$ nos centros de terapia intensiva. Portanto, a prevalência encontrada neste estudo foi maior que aquelas encontradas na literatura.

O tempo de internação total e de internação no CTI superiores a 10 dias, sepse e risco alto e elevado na escala de Braden foram fatores fortemente associados à úlcera por pressão.

Identificou-se o total de 99 úlceras por pressão, localizadas freqüentemente nas regiões sacral $(36,0 \%)$ e calcânea $(22,0 \%)$, sendo que $57,0 \%$ do total de úlceras eram de es-

\section{REFERÊNCIAS}

1. National Pressure Ulcer Advisory Panel. Pressure ulcers prevalence, cost and risk assessment: consensus development conference statement. Decubitus. 1989;2(2):24-8.

2. Amlung SR, Miller WL, Bosley LM. The 1999 National Pressure Ulcer Prevalence Survey: a benchmarking approach. Adv Skin Wound Care. 2001;14(6):297-301.

3. Rogenski NMB, Santos VLCG. Estudo sobre incidência de úlceras por pressão em um hospital universitário. Rev Lat Am Enferm. 2005;13(4):474-80.

4. Almeida Filho N, Rouquayrol MZ. Epidemiologia e saúde. 6a ed. Rio de Janeiro: MEDSI; 2003.

5. Lahmann NA, Halfens RJ, Dassen T. Pressure ulcers in german nursing homes and acute care hospitals: prevalence, frequency, and ulcer characteristics. Ostomy Wound Manage. 2006;52(2):20-33.

6. Gunningberg L. Risk, prevalence and prevention of pressure ulcers in three Swedish health-care settings. J Wound Care. 2004;13(7):286-90.

7. Chan EY, Tan SL, Lee CK, Lee JY. Prevalence, incidence and predictors of pressure ulcers in a tertiary hospital in Singapore. J Wound Care. 2005;14(8):386-8.

8. Allman RM, Goode PS, Patrick MM, Burst N, Bartolucci AA. Pressure ulcer risk factors among hospitalized patients with activity limitation. JAMA.1995;273(11):865-70.

9. Donovan WH, Garber SL, Hamilton SM, Krouskop TA, Rodrigues GP, Stal S. Úlceras por pressão. In: Delisa JA. Medicina de reabilitação. São Paulo: Manole; 1992. p. 553-70. tágio II, ou seja, superficiais, cuja perda parcial da pele envolve epiderme ou derme, ou ambas.

Percebeu-se que as medidas de prevenção ainda não são adotadas de forma sistemática e algumas contrariam as recomendações para uma boa prática clínica, tais como a realização de massagem de conforto nas proeminências ósseas e o reposicionamentos de 6 em 6 horas.

A úlcera por pressão é de difícil tratamento, em geral prolongado e oneroso, o que corrobora a premissa da prevenção. Portanto, diante de pessoas que apresentem riscos para o desenvolvimento de úlceras por pressão, a equipe multiprofissional tem a responsabilidade de implementar medidas preventivas, no intuito de diminuir o impacto desse agravo.

10. Fernandes LM. Úlcera de pressão em pacientes críticos hospitalizados: uma revisão integrativa da literatura [dissertação]. Ribeirão Preto: Escola de Enfermagem, Universidade de São Paulo; 2000

11. Faro ACM. Fatores de risco para úlcera de pressão: subsídios para a prevenção. Rev Esc Enferm USP. 1999;33(3):279-83.

12. Pieper B. Mechanical forces: pressure, shear, and friction. In: Bryant RA, Nix DP. Acute and chronic wounds: current management concepts. $3^{\text {rd }}$ ed. St. Louis: Mosby; 2007. p. 205-34.

13. Redelings MD, Lee NE, Sorvillo F. Pressure ulcers: more lethal than we thought? Adv Skin Wound Care. 2005;18(7):367-72.

14. Ratliff CR, Bryant DE. Guideline for prevention and management of pressure ulcers. Glenview: Wound Ostomy and Continence Nurses Society; 2003.

15. Bruni DS, Strazzieri KC, Gumieiro MN, Giovanazzi R, Sá VG, Faro ACM. Aspectos fisiopatológicos e assistenciais de enfermagem na reabilitação da pessoa com lesão medular. Rev Esc Enferm USP. 2004;38(1):71-9.

16. Medeiros $A B F$, Lopes $C H A F$, Jorge MSB. Análise da prevenção e tratamento das úlceras por pressão propostos por enfermeiros. Rev Esc Enferm USP. 2009;43(1):223-8.

17. Paranhos WY, Santos VLCG. Avaliação de risco para úlceras de pressão por meio da escala de Braden, na língua portuguesa. Rev Esc Enferm USP. 1999;33(1):191-206.

18. Smith LN, Booth N, Douglas D, Robertson WR, Walker A, Durie $M$, et al. A critique of "at risk" pressure sore assessment tools. J Clin Nurs. 1995;4(3):153-9.

\section{Agradecimentos}

Os autores agradecem às enfermeiras auditoras da União dos Médicos de Belo Horizonte (Unimed-BH), pela valiosa participação na coleta de dados.

Correspondência: Flávia Sampaio Latini Gomes Av. Alfredo Balena, 190 - Santa Efigênia CEP 30130-100 - Belo Horizonte, MG, Brasi 